Après la dissection : un dernier mot sur le conte du « Roi-grenouille »

\title{
Conclusion
}

\section{APRÈS LA DISSECTION : UN DERNIER MOT SUR LE CONTE DU «ROI-GRENOUILLE »}

\section{Christiane CONNAN-PINTADO}

TELEM (EA 4195) Université Bordeaux Montaigne christiane.connan-pintado@orange.fr

"What Makes a Repulsive Frog So Appealing"

Jack Zipes

Les études monographiques sur les contes ne sont pas si fréquentes ${ }^{1}$ car les chercheurs abordent plus volontiers de larges corpus en se penchant sur les thèmes ${ }^{2}$, les formes et/ou les enjeux d'un genre - oral ou écrit - qui passionne différentes branches de la recherche depuis deux siècles, précisément depuis que les frères Grimm lui ont accordé une attention d'ordre scientifique. Tel est pourtant le parti adopté par les programmes menés à Clermont-Ferrand au sein du CELIS autour des reconfigurations des contes des Grimm : se saisir d'un conte, en scruter l'amont et l'aval, la production et la réception, les textes et les illustrations, la transmédialité. Après « La Belle au Bois Dormant ${ }^{3}$ » et « Dame Hölle » / « Les Fées ${ }^{4} »$, c’est le KHM 1, « Le Roi-grenouille ou Henri-de-fer », qui a été retenu pour être

1 A l'exception de celles qui se penchent sur « Le Petit Chaperon rouge » et, dans une moindre mesure, sur « Cendrillon ». On signalera cependant, consacré à « La Belle au bois dormant », le beau volume n 289 des Etudes de Lettres de l'Université de Lausanne, Des Fata aux fées : regards croisés de l'Antiquité à nos jours, sous la direction de Martine Hennard Dutheil de la Rochère et de Véronique Dasen, 3-4/ 2011.

2 Voir par exemple les numéros thématiques de la revue Féeries. Etudes sur le conte merveilleux XVII -XIX ${ }^{e}$ siècles.

3 Séductions et métamorphoses de La Belle au bois dormant, sous la dir. de Pascale Auraix-Jonchière et Frédéric Calas, en cours de publication aux Presses universitaires Blaise Pascal, coll. « Mythographies et sociétés », Clermont-Ferrand.

4 Des Fées de Perrault à Frau Holle des Grimm : réécritures et intermédialité, sous la dir. de Dominique PeyracheLeborgne, en cours de publication aux Presses Universitaires de Rennes. 
interrogé dans sa singularité. Pour reprendre quelques-uns des adjectifs déclinés au fil des pages, ce conte si emblématique et si germanique, à la fois stratégique et programmatique, est aussi un conte énigmatique, voire fantomatique car depuis deux siècles il hante les lecteurs, la littérature et toutes les sphères de la représentation, fussent-elles triviales. Germanistes ou non, en provenance de différents pays $^{5}$, européens ou non, tous les contributeurs attestent l'expansion du conte dans l'édition, la littérature et l'imagerie.

\section{Le plus célèbre des contes mal connus}

Catherine Tauveron le rappelle en avant-propos, il s'agit du conte à la fois le plus connu et le plus mal connu, partant d'un conte qui ne cesse d'interroger. Un conte patrimonial qui pourrait correspondre à l'une des définitions du « classique » par Italo Calvino (la $9^{\mathrm{e}}$ sur 14) : « Les classiques sont des livres que la lecture rend d'autant plus neufs, inattendus, inouïs, qu'on a cru les connaître par ouï-dire » (1995, p.10), de ces textes incontournables qui relèvent de la catégorie «LE » de Pierre Bayard : ceux dont on parle sans les avoir lus, parce qu'on en a entendu parler (2007, p. 17). Et qu'on réécrit sans les avoir relus, la profusion de baisers qui ponctue toutes les formes de reformulations en offre l'éclatant témoignage. Si l'on considère la fortune de ce conte, en particulier dans le champ des livres pour enfants, force est de constater « combien la dérivation hypertextuelle sui[t] son cours propre indépendamment de sa source » (Tauveron, 2013, p. 366). Les reformulations déclinées par Marc Parayre attestent la prédominance des produits ludiques qui trivialisent le conte merveilleux en le passant à la moulinette des conventions parodiques : transposition spatiotemporelle, inversion, dégradation. Les représentations du « Roi-grenouille » circulent dans tout l'univers graphique, de la bande dessinée au manga et à la publicité, jusqu'au timbre-poste. Originales ou convenues, ces images reprennent à l'envi les scènes clés qui ont marqué l'imagination, à commencer par celle du baiser, qui n'existe pas. De plus, on peut mesurer à quel point ce conte a réussi à échapper à sa source lorsqu'on se livre au petit jeu qui consiste à questionner quelques moteurs de recherche. Sur le site de la BnF, le catalogue repère 25 titres si l'on précise le nom des auteurs, mais il en fournit 128 lorsqu'on se contente du titre du conte. Sur le site amazon.fr, les Grimm sont crédités de 28 titres, alors que le conte seul fait l'objet de 220 résultats, écart qui entérine le détournement du projet auctorial et rejoint les analyses liminaires de Catherine Tauveron.

5 Dans la mesure où la moitié des contributeurs sont français, la réception française a toutefois été privilégiée. 
Après la dissection : un dernier mot sur le conte du « Roi-grenouille »

Le conte du « Roi-grenouille » a pris place dans les listes du primaire pour le cycle 2 sous la mention « patrimoine ». Etiqueté comme patrimonial par l'institution scolaire, il l'est aussi au sens que Brigitte Louichon (2012) donne à cet adjectif, par sa présence hic et nunc, à travers les nombreux « objets discursifs secondaires » qu'il engendre. Si cette patrimonialité est d'autant moins contestable que le recueil des Grimm a été inscrit en 2005 par l'UNESCO au registre de la Mémoire du monde, on soulignera un peu plus loin la frilosité de l'édition française à l'égard de sa patrimonialisation, frilosité qui affecte aussi l'édition scolaire, comme l'avait montré Hélène Weis dans un article intitulé « La longue absence des contes Grimm dans les manuels scolaires français » (2013) à partir des années 1930. Christine Boutevin et Patricia Richard-Principalli font ici le même constat de la rareté, voire l'absence, du conte dans un corpus de manuels contemporains.

Ce statut particulier du KHM 1 peut justifier qu'une étude monographique se penche sur un conte qui résiste à l'analyse et qui a surtout été étudié dans la sphère germanique et germaniste. Chacun a pu lire, en son temps, l'étude freudienne largement vulgarisée de Bruno Bettelheim, qui lui consacre quelques pages (1976, pp. 352-358), mais les travaux de Jack Zipes sont autrement précieux, en l'espèce son chapitre d'ouvrage (2009) et son article (2008) entièrement dévolus au « Roi-grenouille». Le chercheur germaniste américain doute d'ailleurs que les grilles de lectures freudiennes ou jungiennes (comme celle de Eugen Drewermann) puissent suffire à nous satisfaire et à expliquer l'ascendant étrange que ce conte exerce sur nous. En faisant le point sur les différentes filiations repérées depuis l'Antiquité, Zipes rappelle que les histoires de grenouilles devenues rois sont fort anciennes (il relève une allusion dans le Satyricon $^{\sigma}$ ) et qu'il existe, dans la tradition populaire, des contes dans lesquels il s'agit d'embrasser une grenouille pour s'enrichir car elle se transforme en princesse ${ }^{7}$. En revanche, qu'elle considère le conte sous l'angle anthropologique (François Flahault) ou psychanalytique (René Kaès, Marc Girard ${ }^{8}$ ), la critique en langue française ignore « Le Roi-grenouille » et l'on se prend à regretter que Jean Bellemin-Noël ne l'ait pas pris en compte au prisme de son approche textanalytique lorsqu'il se penche sur Les contes et leurs fantasmes. Il se contente de le mentionner à propos de la grenouille qui annonce

6 Voir l'allusion dans Pétrone, Satyricon, CHAPITRE LXXVII. « Mais, Habinnas, vous étiez présent, je pense, lorsqu’il me dit : "De moins que rien vous êtes devenu un riche propriétaire : [...] moi qui n>étais naguère qu〉une grenouille je suis maintenant aussi puissant qu>un roi" ».

7 Voir W. A. Clouston, « The Story of « The Frog Prince »: Breton Variant, and Some Analogues », (1890), $493-506$.

8 On trouve une unique allusion au « Prince crapaud» dans Les Contes de Grimm. Lecture psychanalytique de M. Girard (Imago, 1990, p. 129), dans un chapitre sur le motif de la fiancée oubliée, sans lien avec le KHM 1. 
la naissance de l'enfant au début de « La Belle au bois dormant » (1994, p. 95).

\section{Des réticences de l'édition française à l'expansion internationale}

Alors que les motifs du «Roi-grenouille » se sont amplement répandus, on ne peut dénier une certaine réticence de l'édition française à l'égard de ce conte, comme à l'égard des contes des Grimm de manière générale, à l'exception des plus célèbres, comme « Blanche-Neige » et « Hansel et Gretel ». Voici l'occasion d'ajouter un volet, dédié au KHM 1, pour compléter l'étude menée antérieurement (Connan-Pintado, 2013) sur l'entrée de ces contes en France à travers la traduction ou l'adaptation. Il faut signaler l'absence notable du « Roi-grenouille » dans les sélections opérées par Marthe Robert en 1955, puis par Jean Amsler, en 1996 - dont les Nouveaux contes visaient pourtant à compléter le corpus antérieurement traduit et publié chez Gallimard. Alors que le conte faisait partie de la Petite Edition (Kleine Ausgabe) destinée aux enfants en 1825, son importation en France reste très limitée au XIX ${ }^{\mathrm{e}}$ siècle. Bien qu'il soit, aux côtés du « Conte du Genévrier », l'un des deux premiers contes des Grimm à être publiés dans la presse au tournant des années $1830^{9}$ (Mathieu, 2007, p. 14), nous n'en trouvons que trois occurrences ${ }^{10}$ dans les huit traductions en volume repérées au cours du siècle. De plus, il n'a pas été retenu dans celle qui fut la plus largement diffusée, celle de Frédéric Baudry chez Hachette en 1855, dont la préface annonçait pourtant vouloir présenter des contes peu connus en France. En revanche, le conte du « Roi-grenouille» s'impose dans les recueils à destination de la jeunesse de la deuxième moitié du $\mathrm{XX}^{\mathrm{e}}$ siècle (huit occurrences sur dix) (Connan-Pintado, 2013, p. 389) et il semble alors être devenu incontournable. Mais c'est en effet dans les recueils qu'il faut aller le chercher, car il fait rarement l'objet d'une publication en singleton ${ }^{11}$.

Non seulement les traductions sont rares au $\mathrm{XIX}^{\mathrm{e}}$ siècle, mais le conte ne figure que de façon sporadique dans les recueils d'adaptations. Par exemple, il est absent des Contes d'un vieil enfant, de Feuillet de Conches en 1858. Mais on le trouve sous le titre « La Grenouille » (pp. 167-173) dans le

9 Cette première traduction, signée N. P., paraît le 4 août 1832 dans le Journal des débats, sous un titre qui élimine le roi-grenouille : « Henri au cœur cerclé de fer ». Elle s'avère pourtant très fidèle au texte source et conserve les deux épisodes qui disparaitront le plus souvent par la suite : le jet de la grenouille contre le mur ainsi que tout le dénouement centré sur le fidèle serviteur. Il en ira de même dans la première traduction en recueil de 1836 par F. C. Gérard (chez Langlumé et Peltier), bien que le serviteur soit ici écarté du titre du conte, devenu simplement « La grenouille ».

10 Il prend place dans la toute première traduction de l'allemand en 1836, par F.C. Gérard, dans un recueil qui ne comporte que 18 contes; dans le volume 2 de la traduction de N. Martin et Pitre-Chevalier en 1848 et dans celle de Max Buchon en 1869 (Connan-Pintado, 2013, p. 373).

11 Il aura malgré tout bénéficié du coup de projecteur donné sur les contes des Grimm par les célébrations du bicentenaire : les éditions Le Genévrier publient en 2013 un bel album illustré par Sara, pour accompagner la traduction d'Armel Guerne. 
Après la dissection : un dernier mot sur le conte du « Roi-grenouille »

recueil que G. Richard dédie à sa fille en 1865, Contes bleus et roses pour l'amusement des grands et des petits enfants ${ }^{12}$. Si les adaptations s'autorisent des broderies peu scrupuleuses ou des réductions marquées par l'édulcoration, certains de leurs détails peuvent comme ici s’avérer intéressants. En effet, la balle d'or y est investie d'une valeur bien supérieure à celle de la matière précieuse qui la compose :

Elle s'amusait à jouer avec une balle d'or que lui avait donné (sic) sa mère \& à laquelle elle tenait beaucoup. Cette balle était marquée de son chiffre, et un vieux devin avait prédit que ce joujou serait la cause de son bonheur. Elle la quittait donc rarement \& la portait dans son sein ou dans sa poche. (p.167)

L'interprétation de Richard rejoint l'intuition de Catherine Tauveron sur le sens de cet objet essentiel au début du récit et lui donne tout son prix : la balle d'or pourrait bien représenter la figure maternelle.

Le prude Maurice Bouchor écarte, en 1912, «Le Roi-grenouille » de sa sélection de Contes d'après la Tradition populaire de divers pays de l'Europe, parmi lesquels figurent pourtant 16 contes des Grimm, mais on retrouve notre conte en 1920 dans un recueil de tout petit format, bien adapté aux petites mains, qui réunit huit Contes de Grimm «adapté (sic) pour les enfants » par Henri Duvernois. Cette délicieuse adaptation intitulée « Le prince grenouille », fourmille de détails qui retiennent l'attention, qu'ils soient poétiques - telle la présence du tilleul animé, motif cher aux Grimm, au début et à la fin du conte - ou humoristiques : ainsi, le personnage titre se nomme Flipperty Flop, onomatopée mimant son sautillement, tout en signalant une influence anglaise. L'adaptation joue avec la source de façon originale, fidèle à la trame et, dans le même temps, émaillée de détails fantaisistes. Pas de baiser ici, ni de projection de la grenouille contre le mur, seulement la tentation de le faire. Quant au fidèle serviteur, personnage majeur du dénouement dans le conte source, il est bien présent, mais sans être nommé. On trouve donc quelques perles dans ces adaptations anciennes, volontiers bavardes et explicatives, comme pour tenter de réduire la part de mystère du conte, et toujours fortement moralisatrices, la fonction pédagogique de la littérature pour la jeunesse l'emportant alors sur sa fonction ludique. Les adaptations ultérieures, au fil de la démocratisation des livres pour enfants, seront plus expéditives et de moins en moins scrupuleuses. Ainsi, en 1938, un an après la sortie du dessin animé Blanche-Neige et les sept nains de Walt Disney, « Le Roi-grenouille » entre dans le recueil intitulé Blanche-Neige par les frères Grimm, chez Nelson, maison d'édition anglo-saxonne à diffusion internationale. Intitulée « La

12 Le titre du recueil souligne sa dette à l'égard de la traduction non autorisée des Contes parue en France en 1824, Vieux contes pour l'amusement des grands et des petits enfants. De plus, l'ouvrage reprend comme elle, et sans les référencer, les gravures de Cruikshank, empruntées à la traduction anglaise de Taylor (1823). 


\section{Christiane Connan-Pintado}

Grenouille enchantée », cette adaptation s'attache avant tout à la personnalité de la jeune, très jeune princesse, stigmatisée comme enfant gâtée capricieuse, égoïste et colérique. Après avoir tenté de se débarrasser de l'animal en le jetant dans un coffre, elle finit par le déposer sur son oreiller de dentelle, et il se transforme aussitôt en un « charmant petit prince, vêtu de satin vert ». On apprend alors qu'il a été transformé par la marraine de la fillette pour la soumettre à une épreuve et vérifier qu'elle n'était pas dépourvue de cœur. Les deux enfants font ensemble « de bonnes parties », car la fillette a appris « à ne plus penser uniquement à elle ». Le mariage est mentionné par convention, mais reporté à plus tard («Quand tous deux furent grands »). On aura noté que la désérotisation de cette version pour jeunes enfants passe par la censure du baiser. Ce type d'adaptation qui ôte toute aspérité au conte continue à circuler de nos jours dans la production éditoriale la moins légitimée.

Pour prendre la mesure de l'empan de la réflexion dans ce numéro d'Ondina/Ondine, on note que la réunion des contributions compose un ample corpus qui s'étend sur deux siècles et englobe aussi bien les différents états du texte publié par les frères Grimm de 1812 à 1857 que ses rééditions, reformulations et réécritures - textuelles et iconographiques - dans un champ qui traverse les frontières du livre de jeunesse. Le conte du « Roi-grenouille » s'enracine dans l'histoire de la littérature européenne et nombre d'histoires fameuses de batraciens sont convoquées passim auxquelles il est tentant de le rattacher, qu'elles soient antiques, comme La Batrachomyomachie - parodie de L'Iliade, à laquelle fait écho le Froschmeuseler (1595) de Georg Rollenhagen qui a pu inspirer les Grimm - ou modernes, à travers divers contes allemands ou écossais, et jusqu'à « La Grenouille bienfaisante » de Madame d'Aulnoy qui préfigure « La Reine Coax » de George Sand. Plus de deux millénaires ont été parcourus de l'Antiquité à la production contemporaine, voyage dans le temps qui est aussi un voyage au cours duquel aucun continent n'est oublié : au premier chef la vieille Europe (France, Allemagne, RoyaumeUni, Espagne, Roumanie), mais aussi l'Afrique avec les albums tunisiens étudiés par Bochra Charnay, l'Amérique, l'Asie et l'Australie avec ceux que présente Sandra Beckett. L'ampleur du champ témoigne de deux phénomènes différents : l'universalité du conte et la mondialisation de la littérature de jeunesse ${ }^{13}$. Le conte du « Roi-grenouille » a essaimé partout où il se publie des livres et Bernhard Lauer, conservateur du Musée Grimm à Kassel, en recense et conserve 105 éditions différentes en

13 Jean Perrot, Mondialisation et littérature de jeunesse, et Du jeu, des enfants et des livres à l'heure de la mondialisaation, Paris, Editions du Cercle de la Librairie, 2008 et 2011. 
Après la dissection : un dernier mot sur le conte du « Roi-grenouille »

provenance du monde entier.

\section{Du texte source aux traductions}

L'édition initiale du conte mérite attention, comme l'avaient déjà observé Ernest Tonnelat en France et Heinz Rölleke en Allemagne, car les frères Grimm le font considérablement évoluer depuis le manuscrit de 1810 jusqu'à la septième édition de 1857. Cyrille François reprend minutieusement ce travail de comparaison grâce à l'outil informatique qui lui permet d'observer, chiffres à l'appui, l'évolution lexicale, syntaxique et stylistique du texte et d'analyser ses incidences sémantiques sur le conte et ses personnages. Loin de s'en tenir à une comparaison entre la première et la dernière édition, il révèle tout le processus de transformation qui ouvre sur le travail même de l'auteur (en l'espèce Wilhelm), étape par étape. Il montre ainsi comment peu à peu les personnages masculins prennent le pas sur la princesse et comment le conte est réorienté vers l'enfance. La part d'équivocité des textes se réduit, pour donner à lire une histoire apparemment plus limpide, dont l'adulte pourra toujours continuer à explorer les méandres, les arrière-plans et les tréfonds sur le mode symbolique, ce que les contributeurs ne se sont pas privés de faire, à l'image des créateurs qui se réapproprient le conte. Le conte relève ainsi du domaine du crossover, analysé par Sandra Beckett, car il s'adresse à deux lecteurs modèles différents, un novice enchanté par la magie d'une histoire de princesse et de métamorphose et un expert mieux armé pour en débusquer le sous-texte. Cette dimension crossover était déjà présente dans le titre initial des Grimm, Contes des enfants et de la maison - même si l'on sait que le recueil initial ne s'adressait pas aux enfants et que la réorientation vers un jeune lectorat ne commence qu'à partir de la deuxième édition. Plusieurs contributions ouvrent pourtant l'empan de ce lectorat dans les œuvres contemporaines, à commencer par celle de Catherine Tauveron qui analyse la transposition poétique d'Anne Sexton à l'aune d'interrogations menées sur le triangle familial de l'inceste - père libidineux, fille abusée, mère absente.

Par le biais de la commande, les éditeurs sont à l'initiative des traductions, des adaptations et des nouvelles illustrations, et l'on pourrait être tenté de réunir sous la même bannière ces approches du conte, mais un tel amalgame est sujet à caution car toute reprise du conte est à questionner au cas par cas. Significativement anonyme dans les productions peu légitimées - à l'exemple de celles qui circulent en Tunisie, étudiées par Bochra Charnay - l'adaptateur se voit contraint de procéder à des réductions liées à l'âge du destinataire et/ou au contexte spatio-temporel et culturel de la réception. 
Tout au contraire, Martine Hennard Dutheil de la Rochère considère le traducteur comme un auteur, en tant que premier lecteur et réécriveur, aussi privilégie-t-elle le terme de transcréation pour désigner l'opération effectuée par celui qui assume la subtile et redoutable responsabilité de créer un nouveau texte en inventant les moyens propres à favoriser le passage d'une langue à l'autre, d'une culture à l'autre. Quant à l'illustrateur, lorsqu'il s'agit d'un véritable artiste, ses images peuvent à leur façon reconfigurer le conte et conduire à renouveler sa lecture.

Les deux études diachroniques qui se penchent sur les traductions du KHM 1, au Royaume-Uni et en Roumanie, font apparaître, selon la formule de Muguras Constantinescu, un certain « brouillage culturel » induit par la circulation des textes en Europe. On sait que la toute première traduction, celle d'Edgar Taylor, en 1823, a exercé une influence considérable, inspiré aux Grimm l'idée d'un volume spécifiquement destiné aux enfants, et modelé la réception ultérieure du recueil. C'est en effet Taylor qui a créé d'emblée, par sa traduction, un contexte de romance, prélude au fameux baiser, absent chez lui mais qui fera florès par la suite. Deux siècles plus tard, la traduction de Philip Pullman, l'un des plus célèbres auteurs contemporains britanniques pour la jeunesse, révèle à quel point l'œuvre des Grimm est patrimonialisée dans la sphère éditoriale anglo-saxonne, où elle reste omniprésente et vivante, contrairement à la France où nulle commande récente d'éditeur n'a permis d'actualiser la réception de ces contes lors des célébrations du bicentenaire.

\section{Conte troublant, lecteurs troublés}

L'une des énigmes persistantes du KHM 1 concerne le personnage du serviteur Henri-le-Ferré, présent dans le titre, absent du conte, et surgissant au dénouement pour y prendre la première place. Deux auteures se sont employées à percer ce mystère et à étoffer le personnage, en fouillant le texte même des Grimm ou ses dérivations traductives et iconographiques. A partir des états du texte et de quelques illustrations, Corona Schmiele mène une enquête aussi palpitante que savamment orchestrée pour donner consistance au serviteur et le tirer l'ombre - lui qui apparaît comme l'ombre même du Roi-grenouille. Ses hypothèses se tournent vers une interprétation autobiographique ou métanarrative : il pourrait s'agir d'une représentation du fidèle quasi jumeau Jacob, ou encore d'un avatar de Wilhelm lui-même, figure cryptée de l'auteur en cocher, présence tutélaire invisible et agissante, à la fois indispensable et en retrait, pour faire avancer le conte. De son côté, Martine Hennard Dutheil de la Rochère montre comment Pullman s'attache à conférer à Heinrich une place majeure dans « un récit 
Après la dissection : un dernier mot sur le conte du « Roi-grenouille »

exaltant l'amour fraternel et la solidarité masculine ». Cette prédilection pour l'amitié entre hommes nous remet en mémoire les valeurs d'une culture homo-sociale venue du fond du moyen âge, comme en écho à l'extrême amitié qui unissait, par exemple, Roland et Olivier entre lesquels Aude - comme la princesse de notre conte - ne pesait guère.

Pour sonder les réactions des jeunes lecteurs face aux énigmes du $K H M$ 1, Pierre Sève a fait lire à des élèves de CM2 ce conte que l'institution destine aux enfants de cycle 2. Le recueil de leurs propos ne manque pas d'intérêt car il permet d'accéder à la réception du texte par des écoliers, discrètement guidés par l'adulte qui accueille et confronte leurs discours. Les étonnements manifestés et les interrogations soulevées recoupent ceux des chercheurs devant le geste violent de la jeune fille, l'intrusion d'Henri-le-ferré à la fin du récit et les relations de ce dernier avec le personnage éponyme. Stimulés par la résistance du texte qui leur offre un terrain de spéculation et de débat, ils se montrent également sensibles à sa qualité artistique et poétique, et confortent l'idée qu'il convient de donner à lire aux enfants des textes de haute tenue littéraire.

Quant aux images surabondantes autour de ce conte, et incontournables dans l'édition pour la jeunesse - albums, recueils, récits illustrés - leur interprétation peut ouvrir de stimulantes perspectives. Ainsi, l'analyse du frontispice du premier recueil traduit en anglais et illustré par Cruikshank attire judicieusement l'attention sur un personnage rieur qui ressemble étrangement à une grenouille et pourrait bien renvoyer au héros éponyme du KHM 1. En comparant les illustrations de plusieurs recueils, Thierry Charnay montre comment les artistes s'attachent aux articulations cruciales du récit dont ils complexifient et enrichissent la lecture grâce à la combinaison des deux systèmes sémiotiques. Le contrepoint des images ajoute parfois au palimpseste une dimension intericonique en révélant l'influence des illustrations historiques sur la production ultérieure, telles celles de Walter Crane qui ont inspiré jusqu'aux albums tunisiens de Bochra Charnay. Les images du « Roi-grenouille » transcendent les bornes du livre de jeunesse dans les albums crossover en provenance du monde entier analysés par Sandra Beckett, ou encore, au-delà de la sphère littéraire, dans les interprétations troublantes des artistes contemporains présentés par Ivana Lohrey.

Une question soulevée régulièrement dans ce volume, inscrit notre conte et sa nombreuse descendance dans une problématique bien en cours aujourd'hui, celle du gender - et c'est sous cet angle qu'Elvira Luengo analyse les résultats de la lecture du conte dans le cadre scolaire et en formation. La réflexion 


\section{Christiane Connan-Pintado}

sur le gender au fil des contributions prend source dans celle du genre grammatical, variable selon les langues, qui conduit à modifier les désignations du (ou de la) protagoniste éponyme et donne lieu à d'intéressantes variations. Grenouille ou crapaud ? Certes, il ne s'agit pas du même animal, mais le genre du mot fait achopper les traducteurs. En allemand, le mot est masculin, comme en grec ou en arabe et les textes traduits par Bochra Charnay respectent cette particularité en usant d'un article masculin pour désigner « le grenouille », mais Catherine Tauveron préfère retenir le terme « crapaud » pour traduire la description - qui soulève le cœur - du père incestueux dans le poème d'Anne Sexton. Ainsi font également les traducteurs roumains étudiés par Muguras Constantinescu :

[...] comme en roumain le terme générique est féminin «broască » ou «broască râioasă » pour rendre " grenouille » et, respectivement, " crapaud », les traducteurs hésitent aussi entre parler du batracien au féminin ou au masculin. Ils choisissent dans le dernier cas la forme « broscoi » qui est, en même temps, le mâle de la grenouille mais aussi un augmentatif, ce qui permet l'association avec le grand batracien qu'est le crapaud, cela d'autant plus que la description d'une bête désagréable dans le conte le permet.

Margaret Hunt, à la fin du XIX siècle, se distingue des autres traducteurs anglais en jouant sur les pronoms : tout au long du récit, elle utilise le neutre pour désigner l'animal - comme il se doit en anglais - mais elle adopte significativement le masculin à partir de la métamorphose. Quant à Bruno Castan, il ose un néologisme masculin, « le grenou », dans sa pièce de théâtre Neige écarlate. Ivana Lohrey fait remarquer la désexualisation ou la sexualisation à l'œuvre dans certaines représentations artistiques, parfois très féministes, mais on a noté une certaine re-sexualisation dans quelques productions pour la jeunesse, comme cette bande dessinée tunisienne où une jeune princesse largement décolletée prend la pose sur son lit auprès d'un crapaud se rengorgeant avec satisfaction. Les audaces ne viennent pas forcément de l'époque contemporaine : George Sand féminise le personnage éponyme dans $L a$ Reine-Coax et Pascale Auraix-Jonchière interroge la féminité de cette « réécriture au féminin » dans laquelle la grenouille est femme - et comme telle aime la parure. En s'appropriant le conte, l'auteure tisse autour de son héroïne un réseau d'instances féminines, contrairement à la plupart des réécritures dans lesquelles la jeune-fille est prise en tenaille entre les deux figures doublement dominantes, masculines et royales, du père et du futur époux. On sait que le conte a été transmis par Dorchen Wild, descendante d'une famille huguenote, et dépositaire d'une tradition dans laquelle s'intriquent des versions européennes d'origines diverses, entre autres celtes. Aussi Jack Zipes se risque-t-il à faire l'hypothèse que Dorchen aurait peut-être été tentée d'adapter le conte (dans lequel la grenouille est une femelle) à sa propre situation, elle qui, ne l'oublions pas, allait épouser Wilhelm quelques années plus 
Après la dissection : un dernier mot sur le conte du « Roi-grenouille »

tard. Certes, la lecture sémiologique et anthropologique du conte par Thierry Charnay, rappelle que les différentes variantes du conte portant sur le fiancé animal représentent la forme la plus accomplie de l'exogamie. Mais à l'heure du mariage pour tous, la question du gender se pose autrement, en particulier dans la conclusion de Martine Hennard Dutheil de la Rochère qui entrevoit une lecture queer du personnage d'Henri-le-ferré : montrant l'étreinte affectueuse de Ranelot et Buffolet, les deux compagnons batraciens d'Arnold Lobel, créés au moment même où l'auteur s'apprêtait à faire son coming out, elle se demande si l'on ne peut voir en eux les descendants du Roi-grenouille et de son fidèle serviteur.

\section{Le dernier mot?}

Pour citer à nouveau Italo Calvino, « la lecture d'un classique atteint son rendement maximum quand on la fait alterner, selon un savant dosage, avec les lectures d'actualité » (p. 12). Telle est bien la proposition du présent volume dans lequel la lecture du texte même des Grimm est combinée à celle de ses innombrables reformulations. Si l'on envisage l'ensemble du recueil des Grimm, on pourra mettre en relation le conte inaugural et le conte final grâce à l'hypothèse stimulante de Cyrille François qui se demande si cette jeune princesse transgressive ne pourrait représenter une figure du lecteur, qui doit s'affranchir d'une certaine éducation littéraire pour se plonger dans la lecture des contes. Faisant écho au dernier conte du volume, « La clé d'or », « Le Roi-grenouille » pourrait alors s'interpréter comme une allégorie de la lecture du livre que le lecteur tient entre les mains.

$\mathrm{Au}$ terme de cette dissection opérée à plusieurs mains, on peut considérer que certains mystères $\mathrm{du}$ « Roi-grenouille » sont devenus un peu moins opaques : Henri-le-ferré a pris quelque consistance et les analyses du texte ou de ses reformulations ont parfois réussi à apporter un éclairage nouveau sur les autres personnages. Prétendra-t-on pour autant avoir eu le dernier mot ? Assurément non, si l'on suit les analyses d'Aurélia Gaillard sur « La clé et le puits », clé des contes et puits sans fond de l'interprétation. La leçon du conte est moins à expliquer qu'à entendre, et l'on espère que « Le Roigrenouille » continuera à nous hanter, comme l'écrit Corona Schmiele qui convoque l'un de ses motifs pour souligner l'impact et le retentissement profond des contes des Grimm sur le lecteur :

Chaque mot tombe dans son âme, comme la boule d'or de la princesse tombe au fond du puits dans « Le roi grenouille ». Il y résonne encore quand l'enfant est devenu un adulte. Incontestablement, les contes des frères Grimm, dans leur littéralité, ont marqué des générations d'enfants, puis d'adultes, et se sont inscrits dans leur conscience où ils résonnent encore. (p. 14) 


\section{Bibliographie}

\section{Adaptations}

Contes de Grimm, coll. Enfantine en couleurs Pierre Lafitte et Cie, adapt. H. Duvernois, 1920.

Contes bleus et roses pour l'amusement des grands et des petits enfants, Librairie du Petit Journal, adapt. G. Richard, 1865.

Contes d'un vieil enfant, de Feuillet de Conches F. S., Paris, Librairie nouvelle, 1858, en ligne sur le site de la BnF : http://gallica.bnf.fr/ark:/12148/bpt6k5623745x

Blanche-Neige par les frères Grimm, adapt. C. et M.-L. Pressoir, ill. E. Barthelemie, Paris, Londres, Edimbourg et Toronto, Nelson Editeurs, coll. « Le coin des enfants », 1938.

\section{Articles et ouvrages}

Bayard, P. (2007). Comment parler des livres que l'on n'a pas lus?, Paris : Les Editions de Minuit. Bellemin-Noël, J. (1994). Les contes et leurs fantasmes. Nouvelle édition revue et augmentée [1983 : PUF]. Montréal : Les éditions Balzac, coll. «L'écriture indocile ».

Bettelheim, B. (1976). Psychanalyse des contes de fées. Trad. F. Ruy-Vidal. [The Uses of Enchantment: The Meaning and Importance of Fairy Tales. New York : Knopf]. Paris : Editions Robert Laffont.

Calvino I. (1995). Pourquoi lire les classiques. Trad. M. Orcel et F. Wahl, [Perchè leggere i classici, Mondatori, 1991]. Paris : Editions du Seuil, coll. « Points ».

Connan-Pintado C. et Tauveron C. (2013). Fortune des Contes des Grimm en France. Formes et enjeux des rééditions, reformulations et réécritures dans la littérature de jeunesse. ClermontFerrand : Presses universitaires Blaise Pascal, coll. « Mythographies et sociétés ».

Drewermann E. (2003). Der Froschkonig. Olten : Walter verlag.

Gaillard A. (2010). La clé et le puits : à propos du déchiffrement des contes et des fables, Féeries, 7 | 2010, 179-192.

Louichon B. (2012). Définir la littérature patrimoniale, Enseigner les classiques aujourd'hui, approches critiques et didactiques, I. de Peretti, B. Ferrier. Bruxelles : Peter Lang, coll. « ThéoCrit' », (pp. 37-49).

Mathieu F. (2007). Deux siècles de traduction des contes des Grimm mais des contes toujours en quête 
Après la dissection : un dernier mot sur le conte du « Roi-grenouille »

de traducteur, dans Nous voulons lire !, 170, 13-20.

Schmiele C. (2015). Masques et métamorphoses de l'auteur dans les contes de Grimm. Pour une lecture rapprochée des textes. Caen : Presses Universitaires de Caen, coll. «Questions ».

Weis H. (201). La longue absence des contes Grimm dans les manuels scolaires français. Ce que cache la forêt des contes..., Les patrimoines littéraires à l'école : usages et enjeux, S. Ahr et N. Denizot. (pp. 195-205). Namur : Presses universitaires de Namur, coll. « Diptyque » .

Zipes, J. (2009). Relentless Progress : the Reconfiguration of Children's Literature, Fairy Tales and Storytelling, Routledge, 2009;

Zipes, J. (2008). What Makes a Repulsive Frog so Appealing: Memetics and Fairy Tales. Journal of Folklore Research, Vol. 45, No. 2 (May - Aug., 2008), (pp. 109-143). Published by: Indiana University Press, Stable URL: http://www.jstor.org/stable/40206971. 